\title{
TEORÍAS DE ENFERMERÍA: IMPORTANCIA DE LA CORRECTA APLICACIÓN DE LOS CONCEPTOS
} \section{TEORIAS DE ENFERMAGEM: IMPORTANCIA DA CORRETA APLICAQÁO DOS CONCEITOS}

\author{
*Mesquita Melo, E., "Lopes, MV de O., "Carvalho Fernandes, AF., "Teixeira Lima, \\ FE., **Barbosa, IV
}

*Doutor/a em Enfermagem. **Mestre em Enfermagem. Universidade Federal do Ceará. Fortaleza-Ceará-Brasil.

Palabras clave: Enfermería, Modelos teóricos, Teoria de Enfermería

Palavras-chave: Enfermagem, Modelos teóricos, Teoria de Enfermagem

\section{RESUMEN}

Investigación teórico-reflexiva cuyo objetivo fue analizar la claridad del concepto estímulo según el modelo de Roy, para mayor comprensión en la práctica de enfermería. Desarrollada en tres etapas: 1.- Levantamiento de estudios que utilizaron el Modelo de Roy; $2^{\text {a }}$ - Lectura dirigida hacia la comprensión del concepto estímulo y 3a - Análisis de la claridad del concepto. Fueron utilizados tres estudios, a nivel de maestrado. El análisis de los estudios demuestra deficiencia en la claridad del concepto estímulo, predominando ambigüedad en la clasificación y diferenciación de los estímulos focales y contextuales, sin consistencia y uniformidad. Se evidencia la necesidad de profundizar en las teorías de enfermería, para su aplicación adecuada en las áreas de investigación, enseñanza y práctica, optimizando y mejorando su uso, además de posibilitar la comprensión de determinados conceptos no suficientemente claros. Las inquietudes surgidas entre los enfermeros podrán motivar un abordaje más profundo de los estudios, posibilitando el avance de las teorías, así como nuevos descubrimientos, dirigiendo los cambios necesarios

\section{RESUMO}

Pesquisa teórico-reflexiva, objetivando analisar a clareza do conceito estímulo no modelo de Roy, para maior compreensáo na prática de enfermagem. Desenvolvido em tres etapas: ia- Levantamento de estudos que utilizaram o Modelo de Roy; 2a - Leitura dirigida para a compreensáo do conceito estímulo e 3a - Análise da clareza do conceito. Foram utilizados tres estudos, em nível de mestrado. A análise dos estudos demonstra deficiencia na clareza do conceito estímulo, predominando ambigüidade na classificacáo e diferenciacáo dos estímulos focais e contextuais, náo existindo 
consistencia e uniformidade. Evidencia-se a necessidade do aprofundamento ñas teorías de enfermagem, para a aplicacáo adequada nas áreas de pesquisa, ensino e prática, otimizando e aprimorando seu uso, além de possibilitar a compreensáo de determinados conceitos, náo suficientemente claros. As inquietacóes surgidas entre os enfermeiros poderáo proporcionar o aprofundamento nos estudos, possibilitando o avanco das teorias, assim como novos achados, direcionando as mudancas necessárias.

\section{ABSTRACT}

Theoretical-reflexive research, whose aim is to analyze the clarity of concept stimulus in the Roy Model, for best comprehension in nursing practice. It was developed in three phases: 1- Search of studies that utilized the Roy Model; 2- Conducted reading for comprehension of the concept stimulus and 3-Analysis of clarity of the concept. The three studies were conducted at master level. The analysis of the studies shows deficiency in the clarity of the concept stimulus, being predominant the ambiguity in the classification and differentiation of the focal and contextual stimuli, without the existence of consistency and uniformity. The need arises to deepen the theories of nursing for adequate application in the areas of research: nursing education and practice, optimizing and improving its use, as well as, enabling the comprehension of determined concepts that are not sufficiently clear. The worries that arise among the nurses will provide a more profound approach to the study, enabling the advance of theories, as well as new findings, leading to necessary changes.

\section{INTRODUCCIÓN}

La enfermería está ganando espacio con la llegada de la investigación y el uso de las teorías, pasando a ser vista como una disciplina importante en el área de salud, además de desempeñar un papel relevante en la promoción, prevención y rehabilitación de la salud.

Las teorías comprenden un conjunto de conceptos y presupuestos, relacionados entre sí, abarcando el campo de la práctica, de la enseñanza y de la investigación. Las teorías de enfermería traen conceptos y proposiciones relacionadas con la enfermería y ligados a una visión del mundo

Las primeras teorías de enfermería se originaron en la década de los sesenta, buscando la relación entre los hechos para el establecimiento de una ciencia de enfermería y una explicación de los acontecimientos referentes al universo natural(2).

Sin embargo, el estudio de las teorías causa todavía alguna incomodidad entre los enfermeros, debido a la inexistencia de una mayor familiarización con el tema. El conocimiento más profundo sobre las teorías y su importancia como aliada en la sistematización de la asistencia propiciaría una reducción en el nivel de expectativa, contribuyendo a la calidad de la asistencia.

Para una exploración más efectiva de las teorías de enfermería, es fundamental la utilización de criterios en cuanto al uso y aplicación, para adecuación al estudio sin distorsiones. Meleis, en su libro Theoretical Nursing, relata el progreso de la Enfermería, con la influencia de presupuestos filosóficos y líneas teóricas. Analiza críticamente y compara las diferentes epistemologías y teorías de enfermería, delineando componentes de la teoría y criterios para la crítica ${ }^{(3)}$.

Es esencial que los enfermeros evalúen las teorías a ser utilizadas, para un mayor auxilio a la práctica y desarrollo de la enfermería, así como para la elaboración de la asistencia de 
forma más sistemática. De ahí la importancia de los modelos de análisis de teorías, buscando el conocimiento más profundo de estas, la reflexión de su utilidad y la contribución a la práctica profesional.

Aunque se perciban dificultades en cuanto al uso de las teorías de enfermería, se observa un mayor número de estudios que utilizan los presupuestos de las teorías para fundamentar los descubrimientos en diversas áreas de la enfermería, entre las que se cita Callista Roy (4: 5 ; 6

El Modelo de Adaptación de Roy conceptúa a las personas como sistemas abiertos, los cuales mantienen interacción continua con sus ambientes, ocurriendo cambios internos y externos. Las personas reciben estímulos constantemente, que exigen respuestas, que pueden ser adaptativas, contribuyendo a la integridad de la persona, o ineficaces, dificultando esa integridad (7).

En el transcurso del modelo, la autora conceptúa el término estímulo como aquello que desencadena una respuesta -el foco de interacción entre sistema humano y ambiente-, originándose en el ambiente externo (estímulo externo) y en el ambiente interno (estímulo interno), siendo descritas tres clases de estímulos que interactúan con las personas: focal, contextual y residual (7).

Los estímulos focales constituyen aquellos estímulos internos o externos más inmediatos al sistema humano. Por ejemplo, la persona puede modificar rápidamente su medio cuando un ruido alto aparece, colocándose en un lugar opuesto al mismo. Estímulos contextuales son todos los otros estímulos presentes en la situación que contribuyen al efecto del estímulo focal, o sea, todos los factores ambientales internos o externos que se presentan al sistema humano, no siendo el centro de la atención, pero que influirán en la manera como la persona reaccionará a los estímulos focales. Estímulos residuales son factores ambientales, internos o externos, cuyos efectos en la situación no están claros o no pueden ser validados.

Vale resaltar que los cambios de las circunstancias pueden alterar el significado del estímulo, o sea, un estímulo puede ser contextual en un momento específico y volverse focal en otro, pudiendo acontecer lo mismo de forma inversa, lo que puede acabar generando dudas en la utilización del modelo, en caso de haber necesidad de definición y caracterización de los tipos de estímulos.

Puede percibirse, frente a la complejidad del modelo, una dificultad en la identificación de los estímulos, por la posibilidad de cambio en su categorización dependiendo de la situación. Generalmente no hay una interpretación única del significado de los estímulos, lo que ocasiona algunas divergencias entre los investigadores y la comunidad científica.

Con el propósito de contribuir a la profundización de las teorías de enfermería y, consecuentemente, a calidad de la asistencia, el estudio tuvo como objetivo analizar la claridad del concepto estímulo, inserto en el modelo de adaptación de Roy, proporcionando mayor comprensión del modelo y de su importancia en la práctica de enfermería.

\section{MATERIAL Y MÉTODO}

Estudio del tipo teórico-reflexivo, realizado en la asignatura Análisis Crítico de las Teorías de Enfermería, del Curso de Doctorado en Enfermería de la Universidad Federal de Ceará, en 2006, buscando delinear la importancia de las teorías en la actualidad, siendo indispensable el análisis reflexivo para la aplicación en campos específicos de la enfermería. 
El desarrollo de la investigación se hizo en tres etapas: 1 - Levantamiento de estudios que utilizaron el Modelo de Roy como fundamento teórico, para la observación del uso del concepto estímulo; 2a - Lectura dirigida a la comprensión del concepto estímulo utilizado por Roy y $3 a$ - Análisis de la claridad del concepto.

Fueron utilizados tres estudios, de nivel de post-graduación (master), que utilizaron el modelo de Roy, siendo que dos tuvieron como objeto de estudio la mujer mastectomizada (4.5) y el tercero, pacientes portadores de hipertensión arterial sistémica(6). A partir de la evaluación de estos, fueron realizados recortes de los trechos del análisis, asociados al Modelo de Adaptación, específicamente los relativos a los estímulos identificados y a la clasificación de estos, para la correlación con lo que está colocado en el modelo, así como la comparación de los abordajes de los estudios, en una búsqueda de puntos comunes.

La 2a etapa del análisis buscó fundamentarse en el pensamiento de la autora sobre el término estímulo y, así, observar la aplicación del término en los estudios, buscando la identificación de divergencias con los conceptos de la autora, así como entre los estudios. La profundización se basó en la publicación sobre el Modelo titulada "The Roy adaptation model" (7).

Para el análisis y la definición de la claridad del concepto (3a etapa), se utilizó como guía el modelo de análisis propuesto por Meleis (3), enfocando el ítem Crítica de la Teoría, en el que se buscó identificar la claridad del concepto estímulo, inserto en el modelo de adaptación de Roy (1).

El modelo de Meleis posee las siguientes etapas: descripción, análisis, crítica, test y teoría de apoyo, compuestas por criterios y unidades de análisis que exploran aspectos de una determinada teoría, con el objetivo de analizar de forma reflexiva su importancia y adecuación a la práctica de enfermería ${ }^{(3)}$.

La crítica es un examen o estimativa de una cosa o situación, estableciendo relación entre estructura y función, observando si la teoría es adoptada por otros. Claridad es tener definiciones operacionales y teóricas, consideradas en la teoría y presentados de manera parsimoniosa, demostrando consistencia con las suposiciones y proposiciones de la teoría (3)

\section{DISCUSIÓN}

\subsection{Presentación de los estudios analizados}

El uso del modelo de Roy en la enfermería (práctica o de investigación) viene siendo contemplado particularmente en trabajos con portadores de enfermedades crónicas. Sin embargo, todavía hay mucho que discutir sobre el modelo, para clarificar los conceptosclave.

\subsubsection{El Modelo de Roy aplicado a mujeres mastectomizadas}

Rodrigues desarrolló la disertación titulada Mujer Mastectomizada: Análisis del Proceso Adaptativo, con el objetivo de analizar la repercusión de la mastectomía en la vida de las mujeres mastectomizadas ${ }^{(5)}$. Durante el análisis, la autora buscó los estímulos vivenciados por la mujer, consecuencia de la enfermedad y de la cirugía, clasificándolos en focales y/o contextuales. 
El estímulo residual no fue explícitamente abordado, a no ser una única vez, representado por miedo a la muerte, aún habiendo situaciones que podrían significar estímulos residuales, como en la declaración: "Yo lo encaro bien (...) a pesar de sentir un cierto complejo. Nunca me expongo, estoy siempre vestida". Observamos vergüenza de la mujer en exponer la mama afectada, aunque usase prótesis mamaria. Se percibe, así, la valorización de la mama como órgano responsable de la belleza femenina afectando al comportamiento, aunque sea de una forma no muy clara.

Los estímulos residuales se encuentran en la situación de una forma implícita, sin ser clara su influencia en los comportamientos de las personas en dicha situación (7).

Podemos enfatizar que el miedo a la muerte representaría más un comportamiento que un estímulo, sin embargo, la autora lo consideró como estímulo, por haber contribuido a una mayor demostración de amor y cariño a los familiares por parte de la mujer. Por otro lado, el miedo está presente en el estudio también como comportamiento, desencadenado por el diagnóstico de la enfermedad y la cirugía, ambos considerados estímulos focales.

Se observa en el trabajo consistencia frente al uso del término, aunque algunas veces esté presente una cierta ambigüedad a lo largo del análisis, en la caracterización de los estímulos. Existe preocupación en la organización y categorización de los estímulos identificados, como en la de los comportamientos asociados, sean adaptativos o ineficaces, así como en la descripción de los modos de enfrentamiento.

Los estímulos focales identificados, en su mayoría, están representados por la enfermedad (cáncer de mama) y la cirugía (mastectomía), implicando también: conflictos en el casamiento y en la formación represora de los padres. Este último representaría un estímulo residual, pues no hay una contribución clara en las respuestas de la mujer, sin embargo fue considerado como focal, tal vez porque en la visión de la autora el comportamiento manifestado por la mujer, representado por la dificultad en expresar sentimientos, fue inmediato. Sin embargo, según el modelo, el estímulo focal tiene un efecto visible en la situación ${ }^{(7)}$, lo que no ocurre en el caso referido.

Los estímulos contextuales contribuyen a las respuestas de los focales(7), siendo representados por la reconstrucción mamaria, ganancia/pérdida de peso, cicatriz quirúrgica, cirugía, uso de prótesis mamarias, edad avanzada, ausencia de compañero sexual, vergüenza del cambio aparente, restricción en las actividades, cambio en el papel de madre, cambios en la relación conyugal, quimioterapia y otras cirugías.

Es visible en el estudio la comprensión sobre los aspectos que deben guiar la clasificación de un estímulo como contextual, mientras que los comportamientos restricción en las actividades y vergüenza del cambio aparente, asociados a la cirugía (estímulo focal) y a la cicatriz (estímulo contextual), fueron clasificados como estímulos, por haber ocasionado tristeza asociada, siendo considerada esta como un comportamiento.

Se observa criterio en la clasificación de los estímulos, basado en el modelo de Roy, pero algunas veces ocurre una inversión de los conceptos, encuadrando un determinado estímulo en otro tipo, así como también clasificando un comportamiento como estímulo. Sin embargo, hay un cuidado continuo en justificar la clasificación de los estímulos, aunque en algún momento de forma no muy clara. 


\subsubsection{El Modelo de Roy aplicado a las familias de mujeres mastectomizadas}

El estudio realizado por Melo abordó el proceso adaptativo de la familia frente a la mastectomía, con el objetivo de identificar estímulos en el contexto familiar de la mujer mastectomizada como consecuencia de la cirugía y conocer los comportamientos de los familiares (4).

Está explícita en la investigación la preocupación por la identificación de los estímulos en la familia, aunque, muchas veces, algunas declaraciones que podrían ser consideradas estímulos pasan desapercibidas, ya que no es fácil distinguir los estímulos y la propia identificación se hace compleja, exigiendo una visión más profunda del modelo y la familiarización con el término.

El diagnóstico de la enfermedad y la comunicación sobre la cirugía fueron colocados generalmente como estímulos focales, pues movilizaron a los familiares hacia la manifestación de comportamientos inmediatos asociados a la situación, siendo citados también: el miedo a la muerte, de metástasis y de la cirugía, el hecho de creer que la enfermedad nunca aparecería en la familia, complicaciones de la cirugía, soledad de la mujer, dificultades financieras, la tristeza de la mujer relacionada con la enfermedad, el miedo de la familia por la recuperación de la mujer y la importancia de la mujer en la familia(4).

El miedo fue clasificado como estímulo, pero esté relacionado con la muerte, metástasis, cirugía o recuperación de la mujer, representa un comportamiento y está claramente evidenciado en el estudio como comportamiento derivado del diagnóstico de la enfermedad y del establecimiento de los tratamientos (estímulos focales).

Como estímulos contextuales percibidos por la autora están: tratamiento quimioterápico, fisioterapia, cáncer, mastectomía, depresión de la mujer, otras enfermedades de la mujer, dificultades financieras, afecto a la mujer, cambio en los roles de la mujer y pronóstico incierto de la enfermedad.

Sentimientos relacionados con la apariencia, creencias sobre autoconcepto y concepto personal sobre cáncer fueron considerados estímulos residuales por afectar a las respuestas, sin efecto claro: "Lloré. Es una mutilación"; "Es la misma persona. Solo porque perdió un seno, no mudó nada"; "Procuramos no tratarla con pena. Tratarla normalmente, para que no se sienta tan pobrecita". Observamos en las declaraciones la valorización de la mama, influyendo de forma indefinida en los comportamientos de las entrevistadas, representando, así, un estímulo residual.

El impacto de la mastectomía es intenso, haciendo que las mujeres y sus familiares, interactúen constantemente con reacciones de miedo: miedo a la pérdida, miedo al cáncer e, inclusive, miedo a la muerte ${ }^{(8)}$.

\subsubsection{El Modelo de Roy aplicado a portadores de hipertensión arterial sistémica}

El estudio de Oliveira tuvo como objetivo investigar el proceso adaptativo de ancianos portadores de hipertensión arterial, frente al envejecimiento y a la necesidad de adherirse a la terapia ${ }^{(6)}$. Los descubrimientos están fundamentados en el modelo de adaptación, aunque, en relación con la claridad del concepto estímulo, no hay uniformidad y fundamento teórico consistente que sustente la clasificación de los estímulos y algunas veces determinados estímulos dejan de ser explicitados. 
Los estímulos focales estuvieron relacionados en su mayoría con aspectos fisiológicos: deficiencia en la capacidad músculo-esquelética e incomodidad respiratoria. El dolor también es citado como focal, como se observa en la declaración: "...este dolor de cabeza es de muchos años, que estoy con él. El día que me ataca es un dolor de cabeza tan grande...". Como comportamientos relacionados con dichos estímulos la autora cita limitación en las actividades físicas e incapacidad para actividades anteriormente desarrolladas.

Por otro lado, el dolor es citado en el mismo grupo de declaraciones, como estímulo contextual, sin justificación para la clasificación: "Era ruin, siempre con dolor de cabeza, este dolor en la nuca... doliendo, y más en esta parte..."; "... comencé a sentir un dolor de cabeza horrible...".

En el Modelo de Roy, el dolor está ejemplificado como estímulo focal, casi siempre visible en el examen físico del paciente (7). En contrapartida, Roy destaca el dolor también como comportamiento no dejando suficientemente clara la diferencia del dolor como estímulo y como comportamiento, pudiendo generar confusión por parte de las personas que utilizan el modelo.

La sensación de pérdida o disminución de la sensibilidad que representaría un estímulo focal, pues exige una respuesta inmediata, representada por el cambio en las actividades diarias, es considerada como estímulo residual, justificada por el historial anterior de accidente vascular cerebral y/o infarto.

Hay una sugestión, durante el análisis, de la enfermedad (hipertensión arterial) como un estímulo focal, desencadenando respuestas ineficaces. Al mismo tiempo la enfermedad, acrecentada por los cambios que conlleva, es explicitada como un estímulo contextual.

Cabe resaltar que un mismo estímulo puede funcionar como focal en un momento y pasar a contextual en otro, dependiendo de la situación enfocada. Alteraciones de las circunstancias en determinada situación pueden mudar el significado del estímulo (7).

Melo estudió los estímulos presentes en mujeres mastectomizadas sometidas a quimioterapia, después de evaluar sus comportamientos, identificando un estímulo focal en determinado comportamiento, que pasa a ser contextual en otro y viceversa(9).

La experiencia de haber abandonado el proceso terapéutico y la muerte del cónyuge son considerados estímulos residuales. Por acumular una experiencia anterior (abandono del tratamiento) y temer las consecuencias, hay una valoración de la terapia para el mantenimiento de la salud y la calidad de vida. La muerte del cónyuge, de acuerdo con las declaraciones, contribuye al sentimiento de aislamiento y soledad.

En otro momento, el envejecimiento y la hipertensión arterial fueron vistos como estímulos residuales, provocando reacciones de miedo e inseguridad, frente a la posibilidad de muerte: "Ahí, yo digo: ¿Muchos años? Muchos años yo no aguanto, ya tengo ese tanto de años, no puedo aguantar mucho..."; "... quien tiene tantos años, no puede esperar muchos más años, sólo si fuese joven...". Es enfatizada una influencia residual de los conceptos emitidos por la familia en la formación del auto-concepto, o sea, el hecho del anciano ser considerado una persona "vieja", contribuye a que formule un concepto semejante, que influirá negativamente en la manifestación de respuestas. 


\subsubsection{Reflexiones sobre los estudios y el Modelo}

Frente a la exposición y comparación de los estudios, se percibe una deficiencia en la claridad del concepto estímulo, dándose, principalmente, ambigüedad en la clasificación y diferenciación de los estímulos focales y contextuales, no existiendo, de una forma general, consistencia y uniformidad.

La claridad de los conceptos está relacionada con la existencia de definiciones operacionales y teóricas consistentes en la teoría y presentadas de forma parsimoniosa(3). La autora levanta la cuestión: ¿Los conceptos están operacionalmente definidos? ¿Parecen tener contenido y construirse válidamente?

Podría haberse considerado en los estudios la definición operacional de estímulo, así como una especificación más clara de los criterios de clasificación, buscando la justificación de los cambios de un estímulo para otro. Meleis considera la necesidad de consistencia, descrita por el grado de congruencia entre los diferentes componentes de una teoría ${ }^{(3)}$.

Cuantos más fenómenos considera la teoría, más ligaciones potenciales tendrá, volviéndose más compleja. La simplicidad de una teoría enfoca menos conceptos y pocas ligaciones para realzar su utilidad. De este modo, podemos clasificar el Modelo de Roy como complejo, por la gama de conceptos, asociados a diversos fenómenos, generando dudas cuando es utilizado en investigaciones científicas ${ }^{\left({ }^{3}\right)}$. Cabe destacar que el término estímulo es repetido a lo largo del modelo, algunas veces de forma innecesaria, representando la tautología, pudiendo contribuir a generar problemas en su comprensión.

Percibimos la necesidad de reorientación en los estudios de determinados estímulos, clasificados de forma ambigua y la exclusión de otros estímulos que, considerando la teoría, corresponden a comportamientos. Destacamos que, algunas veces, en el transcurso del Modelo, la propia autora demuestra ambigüedad, pudiendo ser ejemplificado el dolor, al mismo tiempo citado como estímulo y comportamiento, sin una justificación clara.

Corroborando lo que enfatizan Moura y Pagliuca (10), el uso del método de análisis de teorías de Meleis proporciona una reflexión sobre la significativa contribución de las teorías de enfermería al desarrollo de la profesión, procurando dar respuestas a las necesidades básicas del paciente y estimulando el uso de modelos conceptuales específicos para la profesión.

\section{CONCLUSIONES}

La utilización del modelo de adaptación de Roy presenta validez en los tres estudios, por ofrecer una mejor percepción sobre las situaciones vivenciadas, contribuyendo a la observación de los comportamientos manifestados y auxiliando en las intervenciones de enfermería.

Teniendo en cuenta las divergencias identificadas en los estudios analizados, se hace evidente la necesidad de profundizar en las teorías de enfermería, para la aplicación adecuada en las áreas de investigación, enseñanza y práctica, optimizando y mejorando el uso de las mismas, además de posibilitar la comprensión de determinados conceptos, muchas veces no suficientemente claros.

Las inquietudes surgidas entre los enfermeros que utilizan las teorías de enfermería, podrán proporcionar profundidad a los estudios, posibilitando el avance de las teorías, así como 
nuevos descubrimientos que podrán dirigir cambios necesarios en estas, buscando la consistencia.

\section{REFERENCIAS BIBLIOGRÁFICAS}

1 XIMENES, L.B.; SOUZA, L.J.E.; MOREIRA, R.V.O. A contributo de Wittgenstein para a enfermagem. En: BARRETO, J.A.E.B.; MOREIRA, R.V.O. A decisáo de saturno: filosofia, teorias de enfermagem e cuidado humano. Fortaleza (CE): Casa de José de Alencar/Programa Editorial; 2000. p. 195 - 216.

2 HORTA,V.A. Processo de enfermagem. Sao Paulo (SP): EPU; 1979.

3 MELEIS, A.I. Theoretical Nursing: development and progress. 3rd ed. Philadelphia: Lippincont; 1997.

4 MELO, E.M. Processo adaptativo da familia frente a mastectomia. [Dissertagáoj. Fortaleza (CE): Universidade Federal do Ceará; 2001.

5 RODRIGUES, D.P. Mulher mastectomizada: análise do processo adaptativo. [Dissertagáoj. Fortaleza (CE): Universidade Federal do Ceará; 1999.

6 OLIVEIRA. T.C. Conviver de idosos portadores de hipertensao arterial: avaliagáo do processo adaptativo. [Dissertagáoj. Fortaleza (CE): Universidade Federal do Ceará; 2001.

7 ROY, S.C.; ANDTEWS, H.A. The Roy adaptation model. 2nd ed. Stamford Connecticut: Appleton \& Lange; 1999.

8 SILVA, R,M,; MAMEDE, M.V. Conviver com a mastectomia. Fortaleza (CE): Universidade Federal do Ceará; 1998.

9 MELO, E.M.; OLIVEIRA, T.C.; ALMEIDA, D.T.; ARAÚJO, T.L. Mulher mastectomizada em tratamento quimioterápico: um estudo dos comportamentos na perspectiva do modelo adaptativo de Roy. Rev Bras Cancerol. V. 48, No 1 (2002); p. 21 - 8.

10 MOURA, E.R.F.; PAGLIUCA, L.M.F. A Teoria de King e sua interface com o programa saúde da familia. Rev Esc Enferm USP. V. 38, No. 3 (2004); p. 270-9. 